\title{
Small but effective: potent light-weight additives modulate prenucleation clusters by specific interactions on the molecular level
}

\author{
Patrick Duchstein $^{\$[a]}$, Philipp I. Schodder ${ }^{\$[b]}$, Simon Leupold ${ }^{[b]}$, Thi Quynh Nhi Dao ${ }^{[b]}$, Shifi Kababya ${ }^{[c]}$, \\ Maria R. Cicconi ${ }^{[b]}$, Dominique de Ligny ${ }^{[b]}$, Vitaliy Pipich ${ }^{[d]}$, David Eike ${ }^{[e]}$, Asher Schmidt ${ }^{*[c]}$, Dirk Zahn ${ }^{*[a]}$, \\ and Stephan E Wolf ${ }^{\star[b, f]}$
}

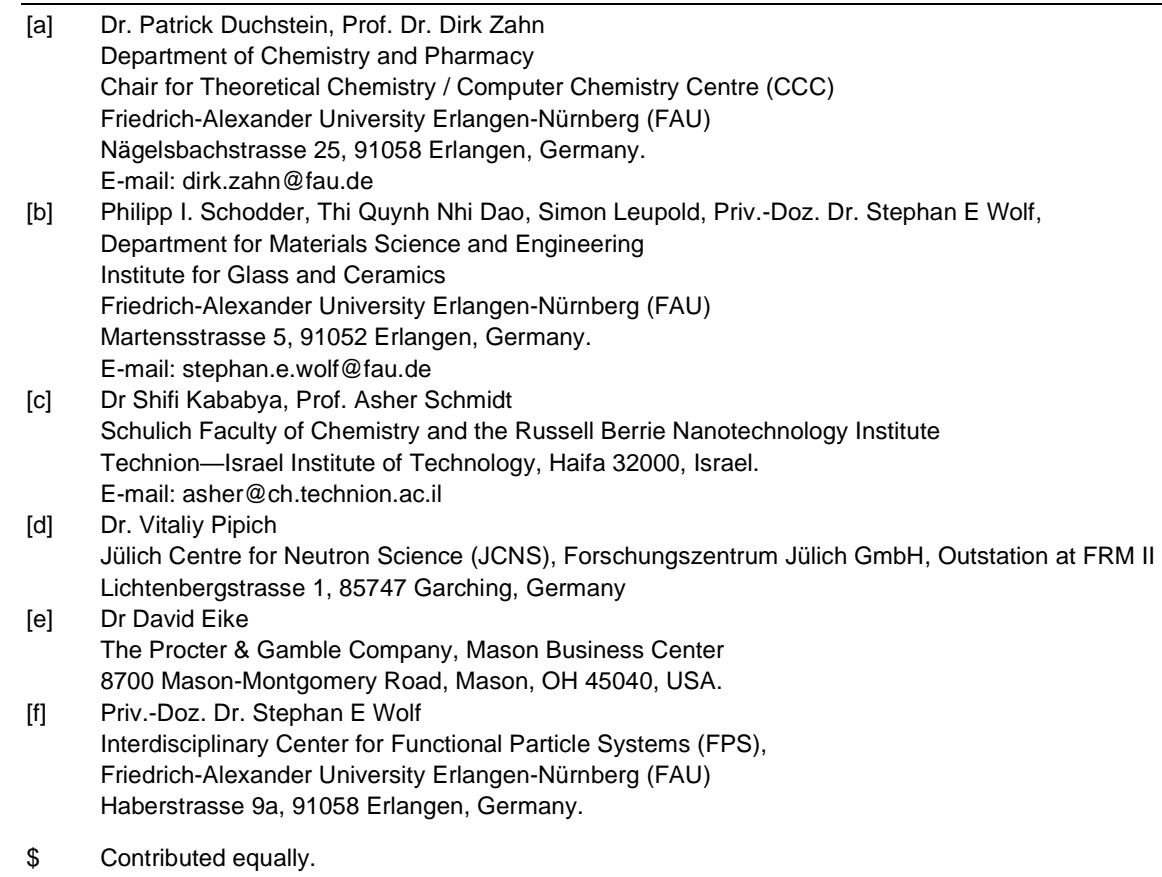

Supporting information for this article is given via a link at the end of the document.

\begin{abstract}
Small-molecular-weight (MW) additives can strongly impact amorphous calcium carbonate (ACC), playing an elusive role in biogenic, geologic, and industrial calcification. Here, we present molecular mechanisms by which additives regulate stability and composition of both $\mathrm{CaCO}_{3}$ solutions and solid ACC. Potent antiscalants inhibit ACC precipitation by interacting with prenucleation clusters (PNC); they specifically trigger and integrate into PNCs or feed PNC growth. Only PNC-interacting additives are traceable in ACC, considerably stabilizing it against crystallization. The selective incorporation of potent additives in PNCs is a reliable chemical label that provides conclusive chemical evidence that ACC is a molecular precipitate derived PNCs. Our results reveal additive-cluster interactions beyond established mechanistic conceptions. They reassess the role of small-MW molecules in crystallization and biomineralization, while breaking grounds for new sustainable antiscalants.
\end{abstract}

\section{Introduction}

The formation of transient disordered phases is characteristic and indicative of kinetically controlled mineral formation mechanisms. Amorphous calcium carbonate (ACC) is the most prominent example as it is a ubiquitous metastable precursor to crystalline biominerals, playing a pivotal role in the biocrystallization process of an overwhelming number of calcifying organisms ${ }^{[1-6]}$ and in the context of pathological biomineralization processes $^{[7-9]}$ Organisms meticulously control ACC formation and transformation by a complex molecular machinery whose actual mechanistic and molecular foundations still require further elucidation ${ }^{[10-12]}$. For example, selected polyelectrolytes are capable of triggering the-still enigmatic-polymer-induced liquid-precursor (PILP) process ${ }^{[13]}$, which yields mineral bodies with atypical morphology via the self-assembly of a liquid-like and highly hydrated transient precursor phase ${ }^{[14-18]}$; it even allows infiltration and coating of complex substrates ${ }^{[19-22]}$.

Since the detection of nanometer-sized stable prenucleation clusters in non-saturated calcium carbonate solutions ${ }^{[23-26]}$, it has been speculated that both formation and structure of amorphous calcium carbonate are intimately linked to these dynamic clusters $^{[24,26]}$. Polyamorphism as a response to changing synthesis conditions is a characteristic feature of $\mathrm{ACC}^{[27,28]}$ Already early on, it was assumed that the correlation between PNC stability, PNCs formation rates, ACC solubility, and ACC near-range order as a function of solution conditions implies that PNCs are precursors of solid ACC ${ }^{[24,26,28]}$. Molecular simulations by Demichelis et al. describe these clusters as solutes in the form of dynamically ordered liquid-like oxyanion polymers (DOLLOP) ${ }^{[26]}$, thus constantly reorganizing networks of 
carbonato-calcium complexes ${ }^{[29]}$; the stability of these coordination clusters appears to be a balance between ionic coordination and ion hydration with entropic contributions ${ }^{[30,31]}$. $\mathrm{THz}$ absorption spectroscopy showed that the formation of solid ACC coincides with a notable change in the dynamic hydrogen-bond network of the solution; it was thus concluded that reduced cluster dynamics induce $\mathrm{CaCO}_{3}$ formation ${ }^{[32]}$. These results have been summarized under the notion of "nonclassical nucleation" ${ }^{[33,34]}$, but this novel view was repeatedly challenged $^{[35,36]}$. For instance, Wallace et al. suggested that DOLLOP formation results from a spinodal demixing of an unstable calcium carbonate solution ${ }^{[37]}$. Up to now, the arguments supporting the idea that PNCs are direct precursors to calcium carbonate are compelling. However, direct chemical evidence was not yet provided as no chemical labeling techniques existed, which would allow tracing their evolution from solution to their final solid state.

Since ACC is a transient precursor to crystalline calcium carbonates $^{[4,38-42]}$, their formation mechanism and its control by additives is a central question for chemistry, biology, geochemistry, and industry. The case of calcium carbonate hemihydrate exemplified that a synthesis route via a metastable phase changes the reaction's energy landscape allowing for the generation of mineral phases that are synthetically inaccessible otherwise ${ }^{[43]}$. Moreover, our limited molecular understanding concerning ACC formation and its ultrastructure considerably impedes the development of sustainable antiscalants. Eutrophic (oligo)phosphonate- and polyphosphate-bearing additives are still in use today since equipotent antiscalants are lacking: although their official phasing out already began in the ' 80 s, the most recent restriction imposed in January 2017 by the European Union concerning the use of phosphor-bearing additives in cleansing agents still allows $0.3 \mathrm{~g}$ of $\mathrm{P}$ per standard dosage ${ }^{[44]}$.

Most scale inhibitors are organic compounds designed to intervene in pre- and post-nucleation stages of calcification. Cölfen et al. have listed up to nine different traits of actions ${ }^{[45]}$, five of which can be qualitatively discriminated by monitoring the solution's calcium activity during $\mathrm{pH}$-constant titrations ${ }^{[24,45,46]}$ while the other four pertain to the properties of the resulting poly(a)morph. Additive-free mineralization profiles yield classic LaMer-curves ${ }^{[24]}$. Deviations from the additive-free mineralization profiles can indicate that the additive (I) removes calcium ions from solution by masking, (II) impacts on prenucleation-stage cluster equilibria, (III) inhibits nucleation and precipitation of a specific secondary phase, (IV) adsorbs to and stabilizes a secondary phase, or (V) favors the subsequent formation of a specific poly(a)morph ${ }^{[45,46]}$. Verch et al. "fingerprinted" common cleansing additives and described, for example, citrate (CIT) as a Type I/II/V additive which leads to a preferred calcite formation and stabilizes PNCs ${ }^{[46]}$. The antiscalant tripolyphosphate (TPP) was classified as a Type I/III/IV/V additive with dominating Type III/V action. Initially, it was concluded that TPP inhibits calcium carbonate precipitation by binding to PNC surfaces while affecting the polymorph selection by adsorption on nucleated particles [45]. Later, it was stated that TPP scarcely interacts with prenucleation clusters since TPP barely influences prenucleation cluster equilibria. It was suggested that TPP instead suppresses nucleation of solid calcium carbonate phases and "just acts"[46] by nucleation and stabilization of a presumed PILP-like phase. These profiling experiments were highly instructive and revealed a remarkable complexity, but the underlying precise mode of molecular interactions between additives and prenucleation solute species remained unclear. The implications of these pathbreaking findings still need to be fathomed further. In applied industrial studies, a simplified model inveterately persists, which dictates that additives are mere "chelants" that interfere with calcium carbonate formation only by calcium sequestration or, utmost, by growth inhibition of calcium carbonate nuclei ${ }^{[47]}$.

In this contribution, we assess the modes by which calcium carbonate solutes molecularly interact with selected small molecular-weight (MW) additives. We conducted precipitation and crystallization experiments along with solid-state nuclear magnetic resonance (SS-NMR) spectroscopy, small-angle neutron scattering (SANS), and differential scanning calorimetry (DSC). We further accompany these analyses with mineralization and $\mathrm{Ca}$-binding profiles. Finally, we correlate these results from in- and ex-situ analyses with molecular dynamics (MD) simulations. We find three different classes of molecular interactions between solutes and small-MW additives, the first is the classically expected binding and masking of calcium ions, effectively reducing $\mathrm{Ca}$ activity. This standard mode of operation leaves the stability of ACC and its composition unaffected. We additionally identified two unforeseen ways by which additives molecularly interact with PNCs. The first of the novel interaction modes is based on the additional association of a few carbonate ions in the second coordination sphere of the additive. This carbonate association also permits intermittent binding of the additive to terminal carbonates of PNCs, with subtle consequences for selected additives such as CIT. Ion-laden additives may incorporate their ionic freight-i.e., calcium and carbonate ions-into the PNC during temporary contact, thereby enhancing PNC growth rates. The second of the novel modes of interaction characterizes the most potent antiscaling agents, e.g., TPP. Additives of this class actively trigger PNC formation by associating a higher number of both calcium and carbonate ions from solution. Due to subsequent cluster overgrowth, these PNCtriggering additives molecularly integrate into PNCs. This incorporation stabilizes PNCs, notably enhancing $\mathrm{CaCO}_{3}$ solubility. We found further that these additives not only impact the solute state but also distinctly alter ACC composition and stability. We found that only those additives are readily traced in the ACC precipitate, individually incorporated in close contact with the amorphous mineral matrix. As an essential consequence of this co-precipitation, $A C C$ is considerably stabilized against phase transformation, both spontaneous and thermally-induced in wet and dry conditions. In combination with SANS, the observed coprecipitation specificity provides chemical evidence that, on a molecular scale, ACC is composed of aggregated PNCs.

Our results demonstrate the central role of PNCs in calcium carbonate precipitation by underpinning PNCs as the molecular building blocks that drive ACC formation. We identified two molecular interaction modes between additives and clusters that affect the formation of PNCs, operating outside the framework of established models. They demonstrate that additive-cluster interactions already regulate solution speciation, solution stability, precipitate composition, and precipitate stability even before phase separation. Ultimately, the newly identified interaction mechanisms break ground for designing new sustainable industrial antiscalants and call for a re-assessment of small-MW molecules in controlling crystallization in general and biomineralization in particular. 


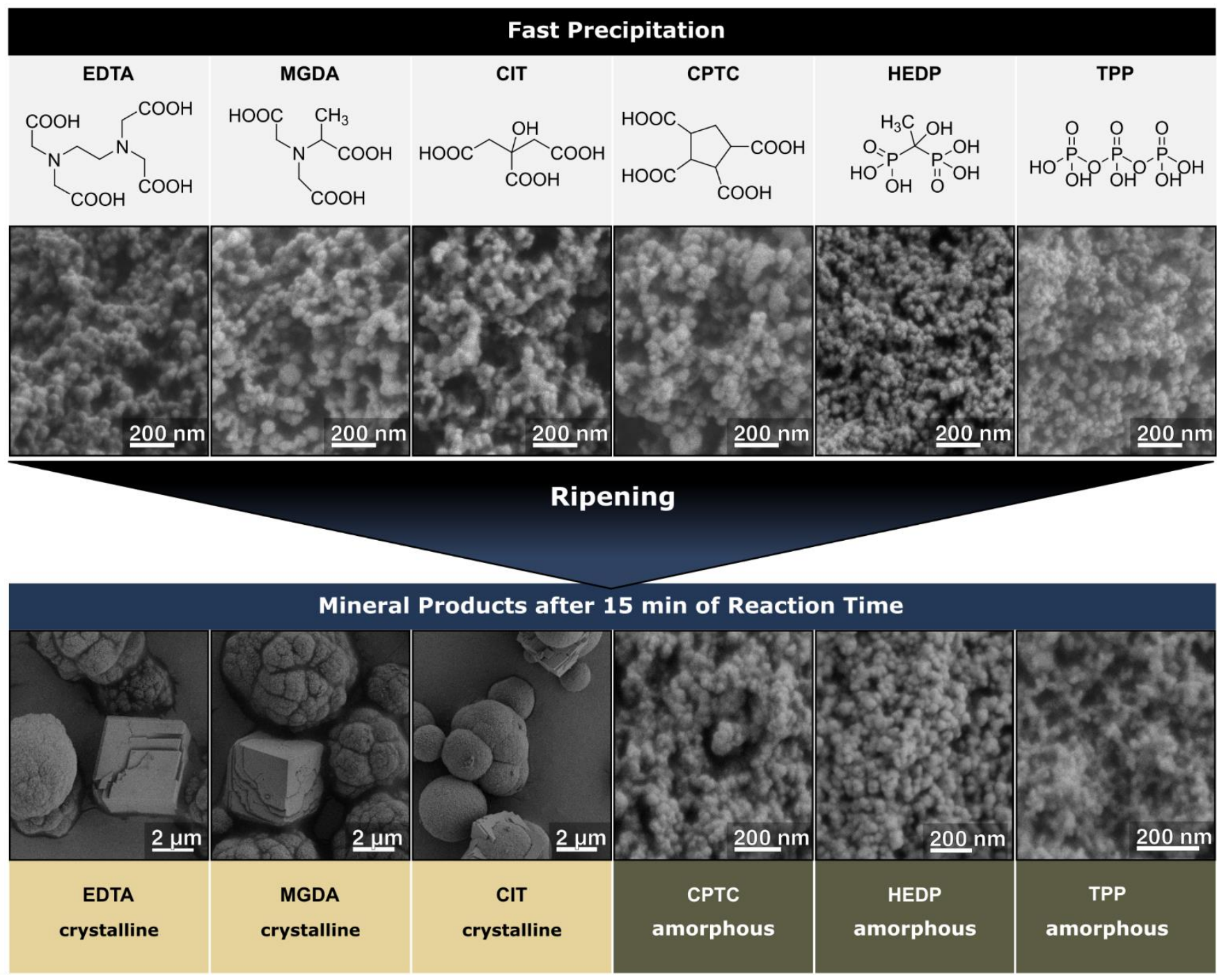

Figure 1. Top-Molecular structures of the employed small-MW additives (For the sake of clearness, the fully protonated version is shown, theoretical speciation at $\mathrm{pH}=9.75$ is given in Table S2B in the SI). Middle - SEM images of the products after fast precipitation in the presence of additives. All powders are amorphous. Bottom - Products retrieved after 15 min of ripening. Powders generated with EDTA, MGDA, and CIT transformed into mixtures of calcite and vaterite. Only in the presence of CPTC, HEDP, and TPP the powders remained amorphous.

\section{Results \& Discussion}

To screen the impact of small-MW additives on the formation of ACC, we selected additives which are all commonly used in cleansing agents as antiscaling additives (Figure 1). Four of these bear carboxylate moieties whereas the other two are characterized by phosphonate or phosphate groups. Ethylenediaminetetraacetic acid (EDTA) is the most commonly employed agent to mask cations from solution, but it shows unfavorably slow biodegradation rates. Methylglycinediacetic acid (MGDA, also known as a-ADA), another masking agent, is a derivate of EDTA with enhanced biodegradability. Further, citric acid (CIT) and cyclopentane-1,2,3,4-tetracarboxylic acid (CPTC) were analyzed; low dosages of the sterically rigid CPTC $\left(0.01-0.1 \mathrm{mg} \mathrm{L}^{-1}\right)$ have been reported to reduce calcite growth rates, whereas the linear and flexible polycarboxylate CIT causes no growth deceleration at identical conditions ${ }^{[48]}$. As commonly used representatives of phosphorus-bearing additives, we further analyzed the bisphosphonate etidronic acid (1-hydroxyethane 1,1-diphosphonic acid, HEDP) and sodium tripolyphosphate (TPP, also known as STP). These additives are largely deprotonated under the given $\mathrm{pH}$ conditions; the additives' dissociation constants and theoretical speciation are provided in Tables S2A and S2B in the supplemental information (SI).

For a first assessment of the additives' impact on ACC formation and transformation, we precipitated ACC in their presence and the absence of additive as a reference. ACC was generated by mixing solutions of calcium chloride $(3.5 \mathrm{~mL}$, $80 \mathrm{mM})$ and sodium carbonate $(50 \mathrm{~mL}, 80 \mathrm{mM})$ at $\mathrm{pH} 9.75$ followed by immediate retrieval of the insoluble precipitate by filtration, thus without further reaction time. These precipitation experiments were conducted at a fixed additive concentration of $800 \mu \mathrm{M}$. In all cases, ACC with similar particle morphologies formed (Figure 1; the reference is given in Figures S1 and S2A).

To examine the stability of the initially formed ACC against phase transformation, i.e., crystallization, we conducted ripening experiments by expanding the reaction time, thus, by retrieving the precipitate from the reaction solution after stirring for additional $15 \mathrm{~min}$. In additive-free ACC (reference) and EDTA-, MGDA-, or CIT-ACC, the initially observed ACC transformed to varying mixtures of crystalline vaterite and calcite. Rietveld refinement analyses showed that additive-free reference experiments predominantly formed vaterite $(97.9 \%)$ besides 
traces of calcite. Vaterite also formed preferentially, though to a lesser extent in the presence of EDTA, MDGA, and CIT $(95.5 \%$, $97.8 \%$, and $85.1 \%$, respectively; Figure S2B). In stark contrast, ACC synthesized in the presence of CPTC, HEDP, or TPP withstood phase transformation, demonstrating a remarkable stabilization of the amorphous state. It is worth noting that a comparable phase transformation behavior is found when nonripened and dried ACC powders were aged by re-suspending in pure water for $15 \mathrm{~min}$. In these aging experiments, calcite dominated in the additive-free ACC (76.8\%), whereas EDTA-, MDGA-, and CIT-ACC mainly formed vaterite $(81.8 \%, 81.1 \%$, and $63.4 \%$, respectively; Figure S2C). ACC precipitated in the presence of CPTC, HEDP, or TPP remained amorphous.

Differential scanning calorimetry (DSC) was performed to quantify the additives' stabilization effects on non-ripened ACC powders (Figure S3). EDTA and MGDA left the crystallization

\section{CPE}

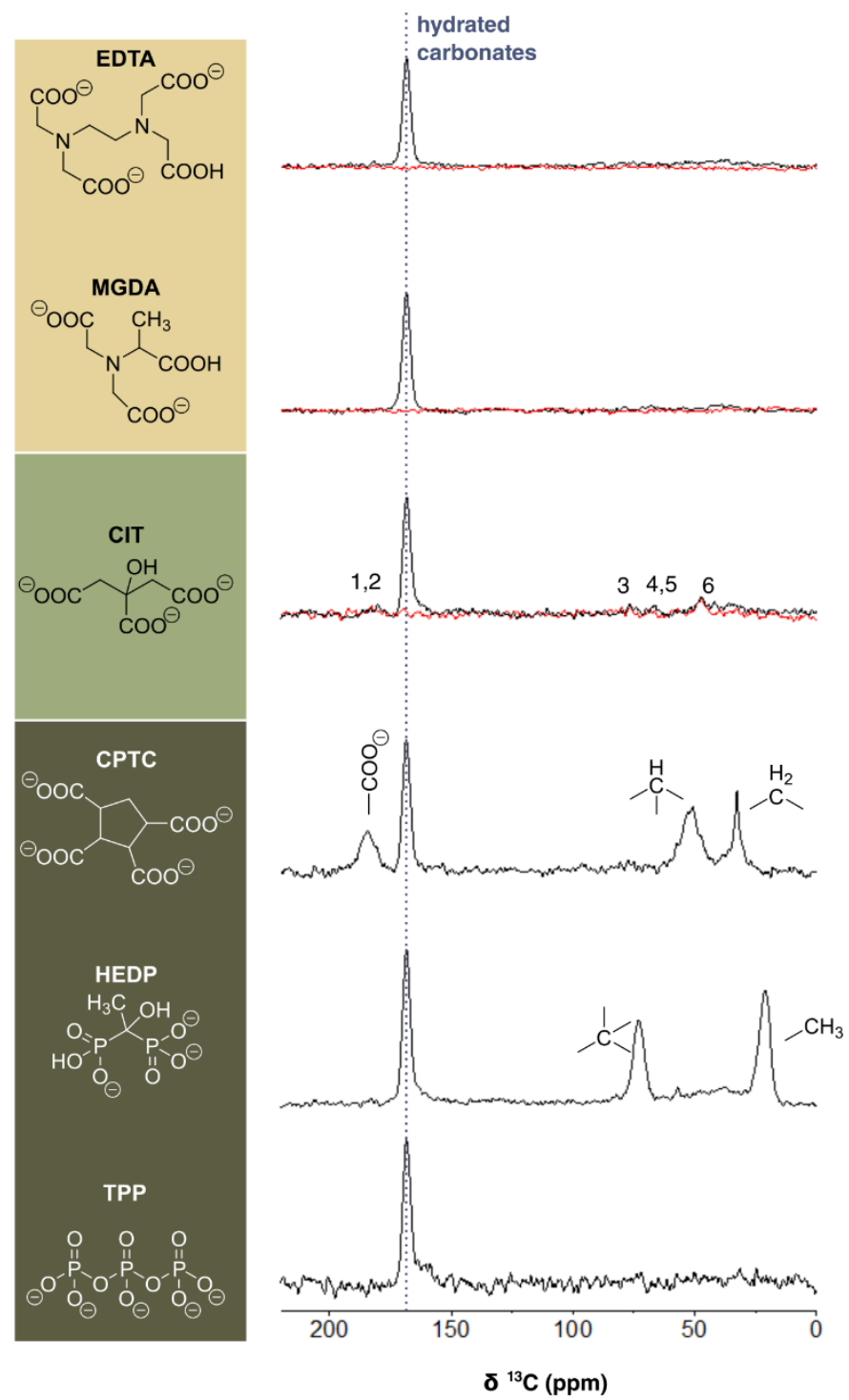

temperature mostly unaffected (approx. $340^{\circ} \mathrm{C}$, matching additive-free ACC). CIT-ACC showed a minor increase in the crystallization temperature to about $360^{\circ} \mathrm{C}$, in agreement with earlier reports ${ }^{[49]}$. Again in striking contrast, the additive triad of TPP, CPTC, and HEDP increased the crystallization temperature considerably (all $>400^{\circ} \mathrm{C}$ ). CPTC led to a crystallization temperature of $411^{\circ} \mathrm{C}$, whereas the most extreme cases were HEDP and TPP, for which no crystallization was observed up to $500^{\circ} \mathrm{C}$.

Small-MW additives exhibit strongly diverging effects on the stability of ACC, although comparable in their molecular size. This differing impact raises the question of whether distinct interaction modes on the molecular level cause this contrasting behavior. One may assume that such a difference in molecular interactions induces a change in ACC composition or structure. We assess this hypothesis in the following paragraphs.

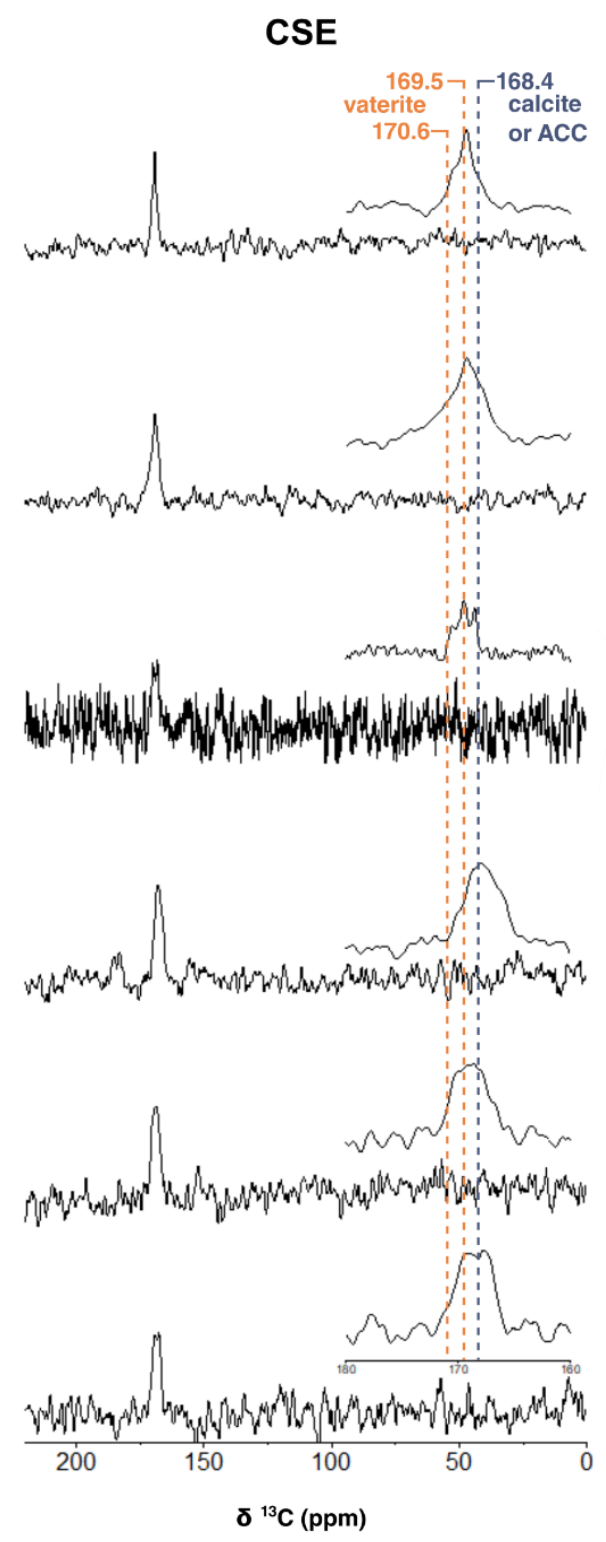

Figure 2. 75.4 MHz ${ }^{13} \mathrm{C} \mathrm{CP}$ (left) and DE (right) MAS SS-NMR spectra of the ACC samples. The additive species drawn are the left are the most abundant at the given $\mathrm{pH}$ of 9.75 (see Table S2B for more details on additive speciation). The CP MAS technique enhances peaks of carbons proximate to hydrogen atoms and as such selectively exposes environments near water and/or the organic additive molecules. The DE MAS technique directly excites all ${ }^{13} \mathrm{C}$ species under quantitative conditions (less sensitive) which allows to identify occurrence of crystalline polymorphs and the relative abundance of additives vs. carbonates. The red overlayed CP MAS spectra for EDTA, MGDA and CIT re-acquired after one to two weeks (following the recording the DE spectra on the right) show vanishing of the carbonate $\mathrm{CP}$ peak, hence indicating complete dehydration as a result of the spontaneous amorphous-to-crystalline transformation. 
Solid-state NMR (SS-NMR) measurements of non-ripened ACC powders enabled us to identify the additives that coprecipitated with ACC, their incorporation levels, and their dispersion in the calcium carbonate matrix. Furthermore, SSNMR served to assess the temporal stability of these precipitates under dry conditions. The notable additive peaks in the ${ }^{13} \mathrm{C}$ and/or ${ }^{31} \mathrm{P}$ cross-polarization (CP) MAS spectra of the precipitates (Figure 2, left and Figures S4a, b, d) show that only HEDP, TPP, CPTC co-precipitated with ACC at levels of approx. 2-3 mol\% (vide infra). The much weaker CIT peaks indicate its coprecipitation occurred at a much lower level. Cross-polarization selects and enhances peaks of carbons (or phosphorous) proximate to hydrogen atoms - in our precipitates; these are found on each of the additive molecules and/or on adjacent structural water molecules that hydrate ACC $(\sim 1: 1)$. As such, these structural water molecules render the amorphous carbonates visible in the CP MAS spectra as fingerprinted by the dominant $168.5 \mathrm{ppm}$ peak (Figure 2). To identify that the coprecipitated additives have become occluded within the ACC matrix, we resort to probing "atomic" proximities between the matrix carbonates ${ }^{13} \mathrm{C}$ 's and the ${ }^{31} \mathrm{P}$ 's of HEDP and TPP using ${ }^{13} \mathrm{C}\left\{{ }^{31} \mathrm{P}\right\}$ CP-REDOR measurements (Figure S4d $)^{[50,51]}$. Specifically, we observe extensive attenuation of the ACC peak due to carbonate-P dipolar interactions, identifying that a large fraction of the carbonates are within $<1 \mathrm{~nm}$ from $\mathrm{P}$-atom of HEDP or TPP additives (evident by the steep REDOR build-up curves demonstrated in Figure S4d). This observation is an indication that these additives are molecularly dispersed in the ACC matrix. Their incorporation levels, as reported by the REDOR build-up curves (Figure S4d), are $6 \mathrm{~mol} \% \mathrm{P} / \mathrm{Ca}$, which for $\mathrm{P}_{2}(\mathrm{HEDP}) / \mathrm{Ca}$ and $\mathrm{P}_{3}(\mathrm{TPP}) / \mathrm{Ca}$ translate into about 3 and $2 \mathrm{~mol} \%$ of additive, respectively ${ }^{[12]}$. The broad additive peaks $\left({ }^{13} \mathrm{C}\right.$ peaks of HEDP, CPTC and CIT, and ${ }^{31} \mathrm{P}$ peaks of TPP and HEDP, Figures 2 and S4) indicate heterogeneity of local environments, i.e., of disorder, consistent with these four additives being molecularly dispersed in an amorphous matrix (ACC) ${ }^{[50]}$. Together these NMR measurements portray additives incorporated as dispersed molecules; thus, they are entrapped as individual molecules within the amorphous mineral matrix. Contrastingly, EDTA-ACC and MGDA-ACC show no additive peaks at all in their CP MAS spectra (Figure 2). Our observations suggest that the CIT incorporation level is about an order of magnitude lower than those of TPP, HEDP, CPTC; the incorporation level of EDTA and MGDA, if any, is at least two orders of magnitude lower.

Of the six additives, only the two phosphorous-bearing additives and CPTC yielded stable, hydrated ACC precipitates (as attested by the persistence of the ACC peak in both the CP and DE MAS spectra; not shown). These are also the additives found to be effectively occluded ( $2 \mathrm{~mol} \%$ ) within the amorphous matrix. The above observations allude to the strong interactions between the solutes and HEDP, TPP, and CPTC at the earlier stages.

As structural water molecules (and to a lesser extent the molecularly dispersed additives) render the amorphous carbonates peak visible in the CP MAS spectra, this peak serves as a marker of the temporal stability of these ACC variants under ambient conditions. Indeed, the finding that EDTA-, MGDA- and CIT-ACC precipitates spontaneously undergo amorphous-tocrystalline transformations accompanied by loss of the structural water is evidenced by the vanishing of the hydrated carbonates peak from their respective CP MAS spectra (red traces on the left of Figure 2). The DE ${ }^{13} \mathrm{C}$ MAS spectra (on the right of Figure 2), a complimentary quantitative technique, identify the resulting anhydrous polymorph mixtures. ACC generated in the presence of EDTA and MGDA has undergone incomplete, solid-to-solid spontaneous transformations to anhydrous disordered vaterite $(\sim 85 \%)$ and disordered calcite $(\sim 15 \%)^{[50]}$. CIT-ACC reached a more advanced transformation to a more ordered (narrower peaks) polymorphs mixture (vaterite and calcite). Longer waiting times resulted in further crystallization with increased calcite fraction (not shown). The additive-free sample (4 $\mathrm{d}$ after retrieval) showed it had transformed to vaterite almost entirely, with a small remainder of ACC (Figure S4c; DE and CP MAS spectra, respectively). The extent of transformation of the ACC samples seen in the NMR experiments is like that observed for ripened samples. After longer waiting times, calcite also appeared in the control sample (not shown), an observation in line with Ostwald's rule of stages. The high abundance of vaterite and its persistence suggests it is stabilized due to our precipitation conditions in line with earlier reports ${ }^{[46,50]}$.

Mineralization profiles monitor the solution's calcium activity during $\mathrm{pH}$-constant titrations and provide detailed information about the amount of bound calcium in the pre- and post-nucleation regimes ${ }^{[24]}$. To be comparable with earlier studies and to attain workable profiling of the additives, the concentrations of calcium, carbonate, and additive were reduced by a factor of 8 relative to the precipitation procedures, maintaining their concentration ratio. Comparing mineralization profiles with the additive-free reference profile and the Ca-binding profiles at a constant $\mathrm{pH}$ value of 9.75 allows a qualitative assessment of how additives impact mineralization processes ${ }^{[45,46]}$, see Figure 3 .

The mineralization profiles recorded in the presence of EDTA and MGDA show their efficient Ca-masking capabilities as the mineralization profiles are shifted stoichiometric to the right; they remove virtually all free $\mathrm{Ca}$ ions from the reaction solution until they reach their Ca-binding capacity, binding one calcium per molecule at maximum. After reaching their Ca-binding capacity, their slopes equal the reference; thus, Ca-saturated masking agents give way for Ca-binding by carbonate/clusters, and $\mathrm{Ca}$ binding proceeds as in the absence of additives. Both EDTA and MGDA allow for a higher maximal prenucleation $\mathrm{Ca}$ activity and lead to elevated post-nucleation plateau levels than in the reference. The latter indicates a change in phase selectivity towards more soluble phases.

In contrast, CIT is not a Ca-masking but only a mediocre Cabinding agent on par with carbonate under the given conditions, as evidenced by its Ca-binding profile. The initial slope of its mineralization profile is only slightly lower than the additive-free reference. Later, the slope steepens without reaching the reference slope, an effect that is more prominent at higher CIT concentrations (Figure S5). The nucleation point is right-shifted by $30-50 \mu \mathrm{M}$, less than half the additive nominal concentration. The changes in $\mathrm{Ca}$ activity recorded by profiling experiments evidence that CIT induces change in Ca-speciation. However, the exact changes with respect to the reference system remain less clear: either Ca-binding by CIT competes with Ca-binding in clusters in an independent fashion-as seen in the extreme case of the masking agents-or CIT stabilizes PNCs, as proposed earlier ${ }^{[46]}$. With regard to the maximum $\mathrm{Ca}$ activity reached in profiling experiments, CIT is the only additive which provides no stabilization of the solution against precipitation although changing slopes considerably. 

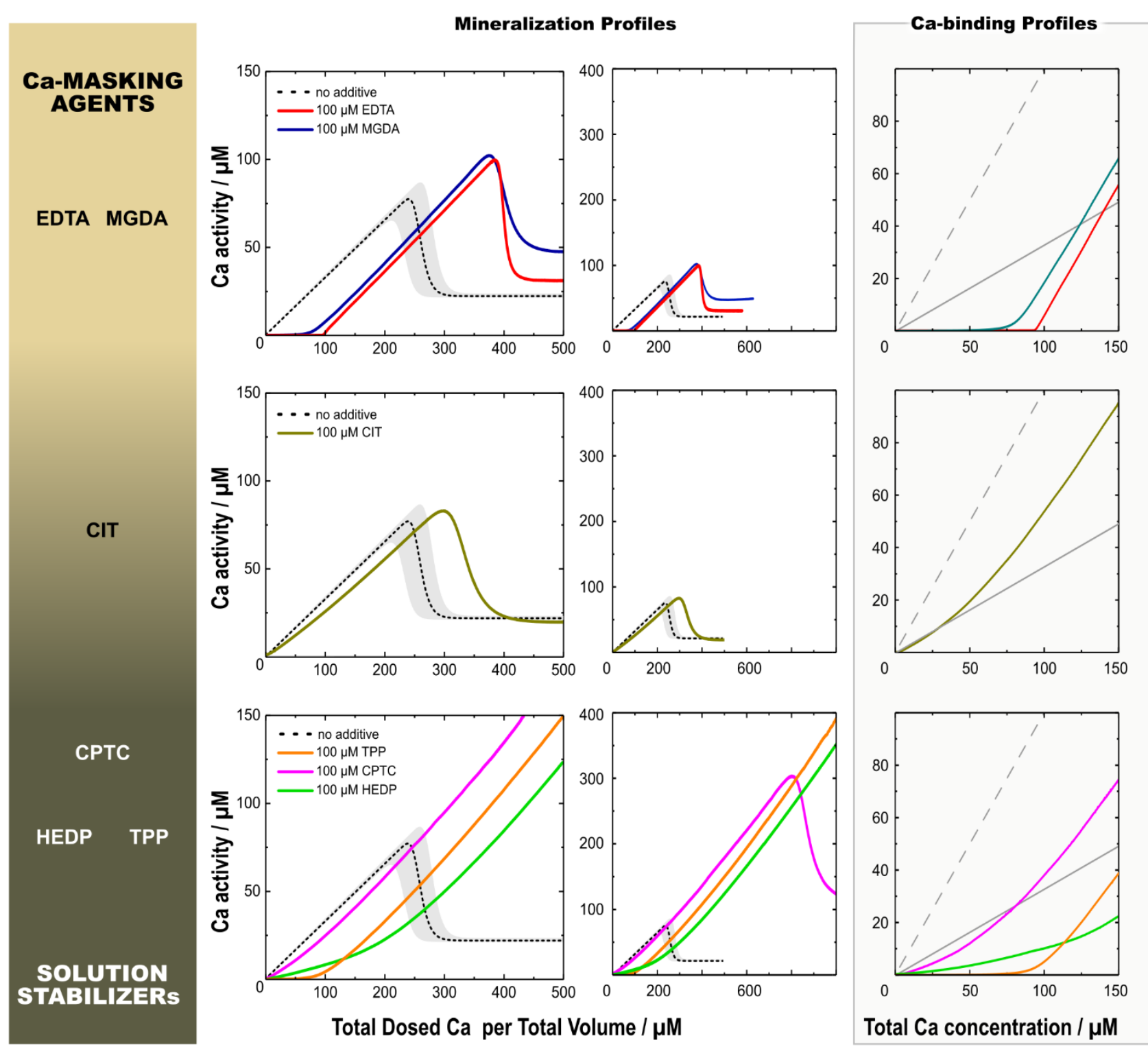

Figure 3. Mineralization and Ca-binding profiles of the individual additives. The mineralization and Ca-binding profiles show the measured Ca activity as a function of the totally dosed Ca per total volume. The dashed black line in the mineralization profiles shows the median of reference experiments, which were conducted in absence of additives but otherwise identical conditions. The gray envelope visualizes the scatter of reference profiles, which is expected due to the stochastic nature of nucleation processes. The dashed gray line in the Ca-binding profiles display the total dosed Ca per total volume, whereas the continuous gray line shows the initial slope of the additive-free reference mineralization profiles. All additives were used at a concentration of $100 \mu \mathrm{M}$; both mineralization and Ca-binding profiles were recorded at a constant $\mathrm{pH}$ of 9.75 .

The remaining triad of additives, i.e., TPP, HEDP, and CPTC, forms a class of its own. They efficiently suppress precipitation by allowing for significantly higher maximal $\mathrm{Ca}$ activities, especially the phosphorous-bearing agents TPP and HEDP. Thus, they act as solution stabilizers, shifting the point of nucleation to the far right. This inhibitory action is independent from a strong Ca-binding activity: CPTC is almost as mediocre as CIT in its Ca-binding affinity in contrast to TPP, which is a Camasking agent as strong as MGDA. Turning to their mineralization profiles, their initial stages resemble their respective Ca-binding curves suggesting that Ca-binding by additives initially outcompetes Ca-binding by carbonate/clusters. In contrast to CIT, the slopes of the individual mineralization profiles approach the reference slope at later stages. Because of this behavior, it was supposed that TPP is "only weakly involved in these [prenucleation] clusters"[46].
Reviewing these results, we can group the inspected additives into two groups of end-members, with CIT as a transitional case. The first group is exemplified by the masking agents EDTA and MDGA. These Ca-binding agents delay precipitation in a stoichiometric manner by reducing the solution's $\mathrm{Ca}$ activity effectively. However, they hardly stabilize the solution against precipitation, i.e., they sparsely increase the maximal $\mathrm{Ca}$ activity reached without precipitation. The triad of solution stabilizers CPTC, HEDP, and TPP form the other set of end members. Their presence exquisitely stabilizes the solution against precipitation. The particular case of CPTC shows that neither strong Ca-binding affinity ${ }^{[46]}$ nor specific chemical moieties such as phosphate or phosphonate groups are necessary preconditions for the solubilizing action of this additive triad.

This grouping corresponds with our compositional analyses and $A C C$ reactivity tests. The masking agents EDTA and MDGA 
are untraceable in the amorphous precipitates; they leave the crystallization temperature of ACC unaffected and exert no stabilization of ACC against crystallization in wet and dry conditions. In contrast, the solution stabilizers CPTC, HEDP, and TPP are dispersed as individual molecules in the amorphous precipitate in significant amounts, and they are closely associated with the surrounding mineral matrix. They impressively stabilize ACC both at wet and dry conditions, increasing the crystallization temperature of ACC considerably. CIT stands out as the intermediate case: it is sparsely traceable in the precipitate, it marginally stabilizes ACC according to DSC measurements, but to an extent too less to considerably delay Ostwald step ripening under dry and wet conditions.

Molecular dynamics (MD) simulations allow us to suggest different modes of actions of the various additives and to establish a link between their behavior on the molecular scale and our experimental observations. We adopted the established simulation model of Gale and coworkers reported in their seminal study of the DOLLOP structure of PNCs ${ }^{[26]}$. We performed an extended series of simulations, exploring the behavior of each additive for $50 \mathrm{~ns}$ in an aqueous solution of $1.5 \mathrm{M}$ calcium carbonate at $\mathrm{pH} \approx 10$ and ambient conditions. By comparing $\mathrm{MD}$ simulations of all six additives, three dynamic interaction modes with PNCs - Classes $\mathrm{A}$ to $\mathrm{C}-$ can be distinguished that originate from distinct ion-association motifs. These three interaction modes are summarized in Figure 4a; close-up snapshots of MD simulation provided in Figure S6 further visualize the different ion association motifs: (A) Additives such as EDTA and MGDA tend to remain dispersed in solution (label $i$ ), whilst associating several calcium ions, and occasionally also few Ca-bound carbonate ions (label ii). These additives do not associate PNCs, thus, they basically associate calcium ions from the parental solution. (B) For additives such as CIT (but to some extent also MGDA) we find the additive to feature association to terminal carbonates that reside at PNC surfaces (label iii). (C) The additive triad of CPTC, HEDP, and TPP associates large numbers of both, calcium and carbonate ions from solution. This behavior triggers PNC formation, and, in the course of cluster growth, these additives integrate into the interior of PNCs.

The complex and dynamic behavior of binding and association of $\mathrm{Ca}^{2+}$ ions from solution is shown for all additives by the three-dimensional plots of occurrence densities provided in Figure S7. These demonstrate that, although $\mathrm{Ca}^{2+}$ ions are preferentially coordinated in specific domains in the surrounding of a given additive, neither the $\mathrm{Ca}^{2+}$ positions nor the terminal oxygen atoms of the acidic groups of the additives are spatially well-defined.

For further quantification and classification of the additive's interaction modes, we evaluated statistics of the nearest-neighbor numbers of $\mathrm{Ca}^{2+}$ ions in the first coordination shell of the additive $\mathrm{n}_{\mathrm{Ca}}$ and the number of nearby carbonate ions $\mathrm{n}_{\mathrm{CO}_{3}}$ in the additive's second coordination shell, indirectly bound to the additives via Ca-bridges. For that purpose, we defined distance delimiters (Table S3) on the basis of the interatomic distance distribution functions between $\mathrm{Ca}^{2+}$ and carboxylate-C or phosphate / phosphonate- $P$ atoms and between carbonates- $C$ and terminal additive oxygens (Figure S8). The variety of complexes observed is reflected by the statistics sampled for the number $\mathrm{n}_{\mathrm{Ca}}$ of $\mathrm{Ca}^{2+}$ ions in the first coordination shell of the additive and the number $\mathrm{n}_{\mathrm{CO}_{3}}$ of $\mathrm{CO}_{3}{ }^{2-}$ ions in the second coordination shell, respectively (Figure S9 and Figure 4a, middle). On the basis of average nearest neighbor numbers $\left(\overline{\mathrm{n}}_{\mathrm{Ca}}, \overline{\mathrm{n}}_{\mathrm{CO}_{3}}\right) \mathrm{a}$ standard Class A agent is observed to accord with $\bar{n}_{\mathrm{Ca}}>\bar{n}_{\mathrm{CO}_{3}}<2$, thus binding calcium ions from the solution but only few carbonates. Additives of type $\mathrm{B}$, which preferably reside at PNC surfaces, must associate about two carbonate ions, whereas additive incorporation into the bulk of the PNCs (Class $\mathrm{C}$ ) is found for $\overline{\mathrm{n}}_{\mathrm{CO}_{3}}>3$. We therefore attribute $\overline{\mathrm{n}}_{\mathrm{Ca}}>\overline{\mathrm{n}}_{\mathrm{CO}_{3}} \approx 2$ and $\overline{\mathrm{n}}_{\mathrm{Ca}}>\overline{\mathrm{n}}_{\mathrm{CO}_{3}}>3$ as characteristics to additive types $B$ and $C$, respectively.

Interestingly, along their time-course, additives may span more than one interaction mode, however the average coordination numbers provide classification means for their preferential mode of action. The coordination number statistics of EDTA, CIT, and HEDP are shown as representative cases in Figure $4 \mathrm{a}$ whereas all profiles are provided in Figure S9. These profiles, along with simulation snapshots (Figure 4a, right), demonstrate that each of the explored small-MW additives exploits two of these three possible interaction modes. For example, MGDA acts both as a Ca-binding agent but occasionally resides at the PNC-surface. Contrastingly, HEDP, TPP and CPTC are predominantly found in the bulk volume of PNCs (label $\boldsymbol{v}$ ) but also, less frequently, at their surfaces (label iv). Indeed, the members of this additive triad are not found to be individually dispersed in the solution, but stay associated with clusters. Simulation snapshots revealed that these three additives actively triggered PNC formation and were subsequently overgrown by PNCs. This classification based on coordination statistics (see also Table S4) lines up with the grouping of additives suggested by our experimental evidence on ACC composition and stability. Together they highlight the key role played by strong additivecluster interactions to produce a potent additive. These interactions provoke additive integration in the PNC and in turn co-precipitation within ACC. As such they play dual roles solution stabilization and precipitated ACC stabilization.

The MD simulations furthermore showed an unforeseen concomitant of the capability of CIT to attach to PNC surfaces. Indeed, CIT interactions to PNCs are found as a dynamic equilibrium of association and dissociation events. In spite of the short time scales of MD runs (50 ns), we consistently find that the number of calcium and carbonate ions associated to CIT additives are larger during CIT attachment than upon detachment from PNC surfaces (see series of snapshots in Figure 4B), hence demonstrating that CIT unloads some of its ionic cargo at the PNC surface. Thus, CIT can be described as a carrier molecule that actively feeds the growth of PNCs by transporting both calcium and carbonate ions to PNCs. 

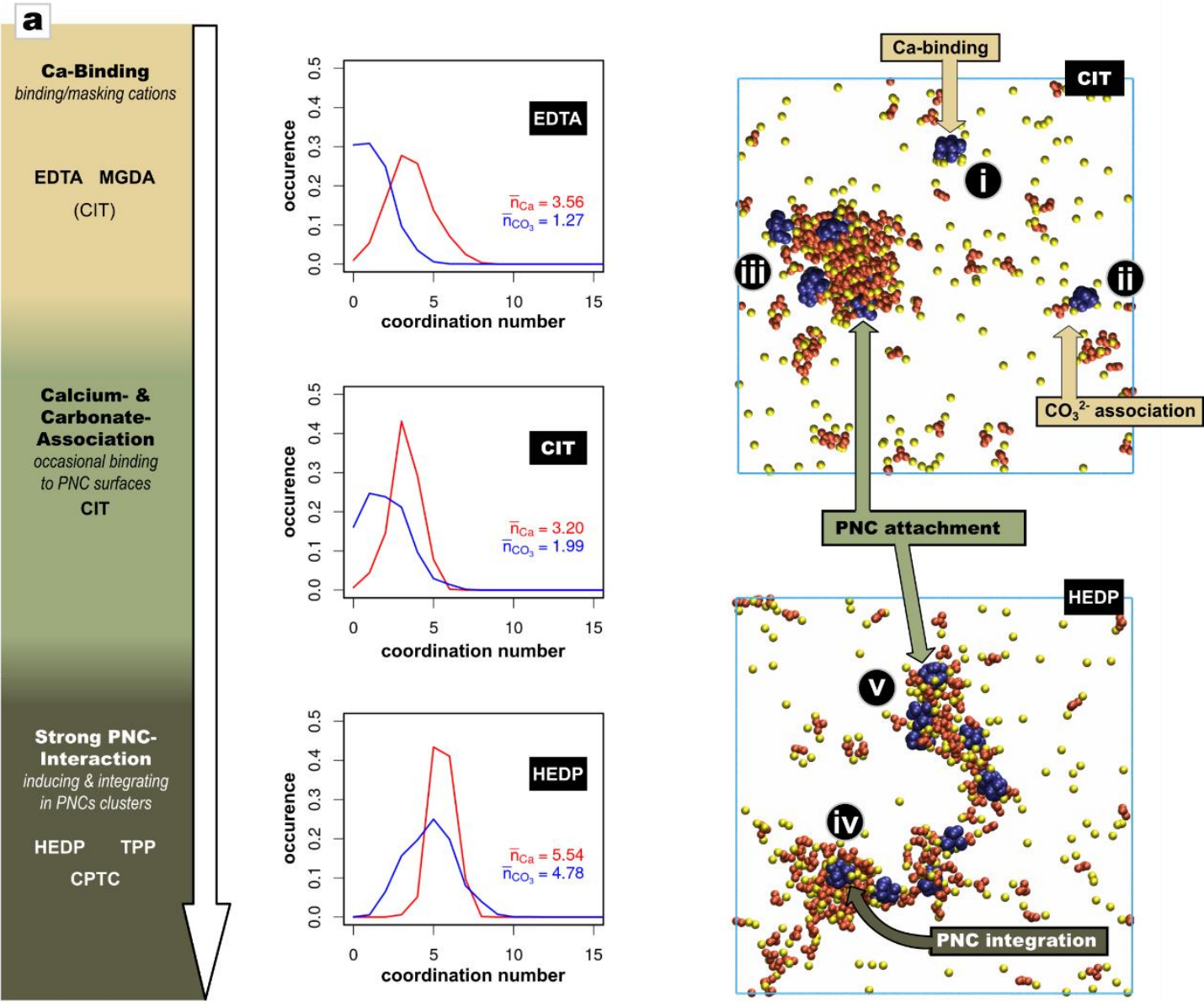

b
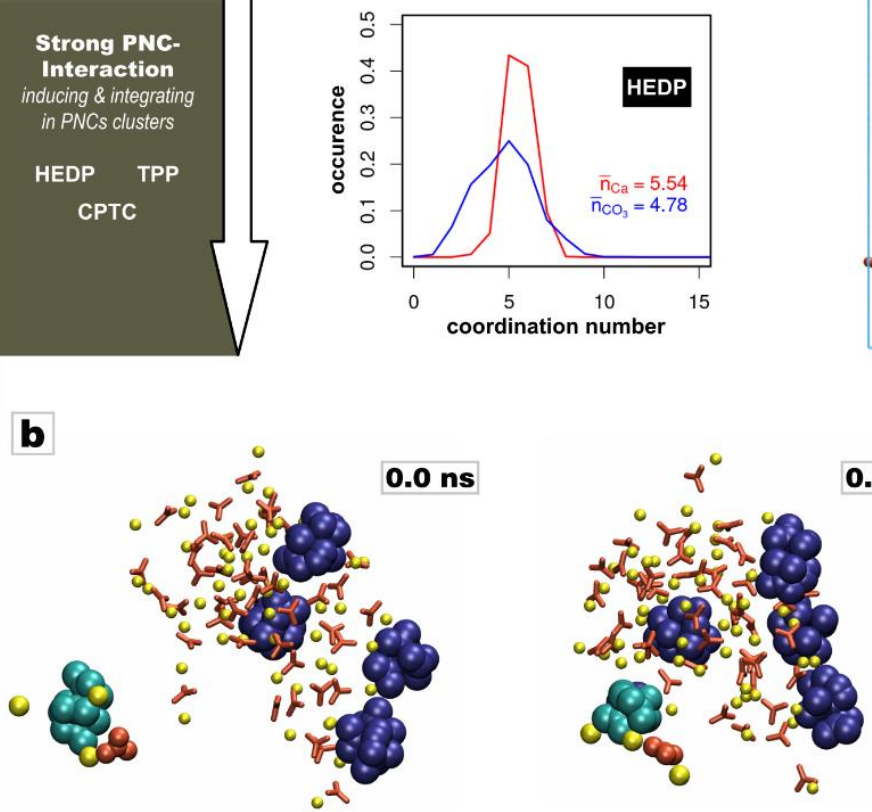

$0.1 \mathrm{~ns}$

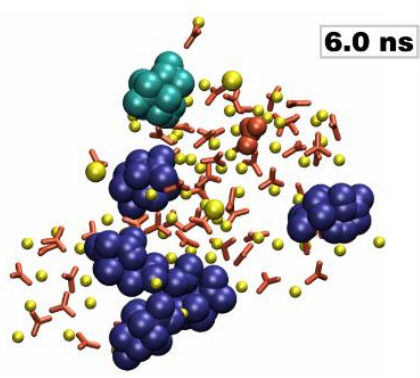

Figure 4. $\mathrm{MD}$ simulations in presence of additives. Additives are colored in blue, $\mathrm{CO}_{3}{ }^{2-}$ ions in red, $\mathrm{Ca}^{2+}$ ions in yellow. water molecules are not shown for the sake of legibility. Snapshots are representative top views of the simulation box. (a) MD simulations provide an additive classification based on the nearest-neighbor numbers of associated $\mathrm{Ca}^{2+}$ ions in the first and $\mathrm{CO}_{3}{ }^{2-}$ in the second coordination shell of the additive. While all additives bind $\mathrm{Ca}^{2+}$ ions, Three distinct modes of $\mathrm{CO}_{3}{ }^{2-}$ association are apparent. Chelating additives such as EDTA bind $\mathrm{Ca}^{2+}$ ions while hardly associating $\mathrm{CO}_{3}{ }^{2-}$ ions. they bind to PNC surfaces only infrequently. The coordination occurrence diagram of EDTA reflects this behavior, showing only limited $\mathrm{CO}_{3}{ }^{2-}$ association numbers (in the center top, $\overline{\mathrm{n}}_{\mathrm{Ca}}>\overline{\mathrm{n}}_{\mathrm{CO}}<2$ ). The occurrence-diagram of CIT differs in this respect (in the middle center, $\overline{\mathrm{n}}_{\mathrm{Ca}} \overline{\mathrm{n}}_{\mathrm{CO}_{3}} \approx 2$ to 3 ), highlighting it as an intermediate case. These simulations shows that CIT features several calcium $\mathrm{Ca}^{2+}$ ions in its near vicinity (see label $\boldsymbol{i}$ in the upper right snapshot of $\mathrm{CIT}$ ) but can also associate $\mathrm{CO}_{3}{ }^{2-}$ ions via $\mathrm{Ca}^{2+}$ bridges (see label ii). CIT's average carbonate association number $\bar{n}_{\mathrm{CO}_{3}}$ has a further contribution since CIT preferentially associates to PNC surfaces, but does not incorporate into PNCs entirely (see label iii). Additives such as HEDP attract larger numbers for both $\mathrm{Ca}^{2+}$ and $\mathrm{CO}_{3}{ }^{2}$ ions $\left(\overline{\mathrm{n}}_{\mathrm{Ca}}>\overline{\mathrm{n}}_{\mathrm{CO}}>3\right.$ ) and incorporate into PNCs (label iv in the lower snapshot of HEDP). Occasionally, they reside at the PNC interface (label $\boldsymbol{v}$ in the lower snapshot). The simulations show that these additives actively trigger PNC formation; thus, PNCs form around the additives and the additive gets overgrown by the forming cluster. (b) Consecutive snapshots taken after $15 \mathrm{~ns}$ of initial equilibration show a single $\mathrm{CIT}$ molecule (highlighted in green) laden with three $\mathrm{Ca}^{2+}$ ions and one $\mathrm{CO}_{3}{ }^{2-}$ ion. It attaches to a PNC, while some other $\mathrm{CIT}$ molecules remain at the surface of the PNC during the sampled time frame. Upon attachment, the PNC incorporates the CIT-associated ions. Later, CIT was found to detach from the PNC surface. 
SANS allowed us to probe the ultrastructure of the nonripened ACC and showed a multilevel structure for all of these powders, be it in the presence or absence of additives (Figure S10). We applied a multilevel Beaucage fit with Porod constants fixed to a value of four for all hierarchical levels, thus with the fundamental assumption of dense and non-porous building units. This approach allowed us to extract comparable gyradius values of the smallest entities (Table S5). For all powders, including the reference sample, we found gyradii ranging from $18.2 \pm 0.2 \AA$ to $29.8 \pm 0.9 \AA$. This size in the small nanometer range correlates well with the estimated size of prenucleation clusters ${ }^{[2,24,52]}$. Thus, SANS shows that all ACC samples, regardless of the presence or absence of additives, show a nanoscopic organization ${ }^{[53]}$ that fits the reported sizes of prenucleation clusters.

\section{Conclusion and Outlook}

Our experimental findings show that only selected additives such as CPTC, HEDP, and TPP significantly stabilize the solution against precipitation (mineralization profiles). These additives also lead to a remarkable stabilization of ACC against spontaneous crystallization in dry and wet conditions and against thermally induced transformation (ripening and DSC). Only these solution-stabilizing additives considerably incorporate into ACC upon precipitation as individually dispersed molecules closely associated with the amorphous mineral matrix (SS-NMR). Common Ca-masking additives such as EDTA and MGDA neither impact ACC stability nor can they be traced in the precipitate. MD simulations on calcium carbonate solutions disclose that only these solution-stabilizing additives induce PNC formation and incorporate into PNCs while additives such as EDTA and MGDA hardly interact with PNCs but stay dispersed in solution.

The strong correlation between PNC incorporation of solution-stabilizing additives in simulation, with their abundance in the resulting ACC precipitate stabilizing it, and the pronounced calcium carbonate solubilization expose for the first time a unified multidisciplinary view on the path of action of high-potency additives. The fact that precipitation is strongly suppressed only in the presence of PNC-incorporating additives demonstrates that solution stability against precipitation and of the ACC precipitate against crystallization are intimately connected via the stability and composition of PNCs ${ }^{[35,46,54]}$. These findings also support the view that PNCs are the fundamental building units of ACC. SANS data provide further corroboration, suggesting that ACC forms by the agglomeration of nanometer-sized units ${ }^{[53]}$ that do not coalesce but preserve their nanoparticular character upon assembly.

In this study, the additive CIT exhibits an intermediate mode of action. Only trace amounts of CIT occur in the precipitates (SSNMR). It marginally stabilizes ACC against thermally-induced transformation (DSC), and no stabilization was observed against transformation in solution (ACC ripening experiments). CIT is the only additive that does not significantly increase the maximal $\mathrm{Ca}^{2+}$ activity (mineralization profiles). Still, CIT alters the solution's Ca speciation and, based on mineralization profiles, it has been concluded earlier that CIT exerts a distinct stabilization effect on $\mathrm{PNCs}^{[45,46]}$. Our MD simulations and mineralization profiling show that CIT does not act as a Ca masking agent. By feeding PNC growth, CIT promotes PNC formation from ion solutions. However, the main effect exhibited by CIT is PNC surface binding. This, we infer, makes CIT to act as a surfactant that reduces PNC-PNC interactions and hinders PNC agglomeration, hence leading to slowed-down precipitation whilst only weakly affecting ACC stability (as only a small fraction of CIT is incorporated in the precipitate).

In conclusion, we classify the modes of action of additives based on their molecular interactions with PNCs (Classes A to C). As additives typically show not only one but two of these traits, our classification highlights the preferential role of an additive. It is the weighted contributions of mechanisms that lead to all of the experimentally observed properties (see Figure 3).

A. The apparent mode of action is the sequestration of cations from solution, i.e., Ca-binding. This trait simply reduces the apparent activity product of the parental solution. Camasking agents such as EDTA and MGDA are relatively clean representatives of this class, masking individual $\mathrm{Ca}^{2+}$ ions. In MD simulations, they also weakly associate further calcium ions in their first coordination shell but less than two carbonate ions in their second coordination shell $\left(\overline{\mathrm{n}}_{\mathrm{Ca}}>\overline{\mathrm{n}}_{\mathrm{CO}_{3}}<2\right)$.

B. The second molecular interaction mode is typified by the preferential affinity of additives to PNC surfaces. In this mode additives associate a small number of calcium and carbonate ions $\left(\overline{\mathrm{n}}_{\mathrm{Ca}}>\overline{\mathrm{n}}_{\mathrm{CO}_{3}} \approx 2\right)$. Then, the additive can bind to PNCs surfaces without fully integrating into the PNC bulk volume. CIT is the additive that mainly takes this mode of molecular interaction. As a concomitant effect, CIT enhances cluster growth by transporting $\mathrm{Ca}^{2+}$ and $\mathrm{CO}_{3}{ }^{2-}$ ions from solution to the PNCs.

C. The third mode of action is shown by additives that generously associate both $\mathrm{Ca}^{2+}$ and $\mathrm{CO}_{3}{ }^{2-}$ ions from solution, thereby provoking PNC formation $\left(\overline{\mathrm{n}}_{\mathrm{Ca}}>\overline{\mathrm{n}}_{\mathrm{CO}_{3}}>3\right.$, e.g., the triad of CPTC, HEDP, and TPP). Their incorporation into clusters leads to a notable suppression of precipitation. They also provide a stabilization of ACC against transformation as these additives are incorporated into the precipitate as individually dispersed molecules.

Based on our results, PNCs can be described as gatekeepers that govern the chemical composition of PNCs and, thereby, the composition of ACC and, ultimately, the macroscopic properties of ACC. The selective incorporation of small-MW additives in PNCs, such as potent class $C$ additives, renders these additives accountable chemical labels that provide the first conclusive chemical evidence that ACC is a molecular precipitate derived from PNCs. Moreover, the molecular interactions of additives with the surrounding PNC/ACC establish the basis for our molecular level insight of the underlying mechanisms responsible for high potency additives.

We expect that the above interaction modes are also valid for other precipitation systems, if prone to amorphous precursor phases and PNCs. Potentially, our work gives new impulses for non-eutrophic, sustainable mineralization inhibitors and for novel drug design concepts against nephrolithiasis and other atopic biomineralization processes. In recent years, several studies have demonstrated the occurrence and importance of small-MW molecules in biomineralization processes ${ }^{[12,55-58]}$. Our findings have substantial implications for these systems, including other bio- and ACC-mediated mineralization processes (e.g., in geosciences), as they highlight their unforeseen but pronounced and molecularly intricate effect on the formation of minerals and amorphous solids. 


\section{Acknowledgments}

PD, DZ, and SEW acknowledge funding from Procter \& Gamble Inc. SEW further acknowledges financial support by an Emmy Noether starting grant by the Deutsche Forschungsgemeinschaft (DFG, German Research Foundation), grant number 251939425. SEW also acknowledges financial support provided by JCNS to perform the neutron scattering measurements at the Heinz MaierLeibnitz Zentrum (MLZ), Garching, Germany. AS and SK acknowledge the Israel Science Foundation grant 2001/17 for their financial support. We also thank Dr Neamul Khansur for his support in XRD analyses.

Keywords: nonclassical crystallization - calcium carbonate $•$ biomineralization $\cdot$ nucleation $\cdot$ antiscalants $\cdot$ solid-state NMR • REDOR

[1] L. Addadi, S. Raz, H. Weinfurter, S. Weiner, Adv. Mater. 2003, 15 959-970.

[2] L. B. Gower, Chem. Rev. 2008, 108, 4551-627.

[3] J. Seto, Y. Ma, S. A. Davis, F. C. Meldrum, A Gourrier, Y.-Y. Kim, U. Schilde, M. Sztucki, M. Burghammer, S. Maltsev, C. Jäger, H. Cölfen, C. Jager, H. Cölfen, Proc. Nat. Acad. Sci. USA 2012, 109, 1-6.

[4] T. Mass, A. J. Giuffre, C.-Y. Sun, C. A. Stifler, M. J. Frazier, M. Neder, N. Tamura, C. V. Stan, M. A. Marcus, P. U. P. A. Gilbert, Proc. Natl. Acad. Sci. 2017, 114, E7670-E7678.

[5] Y. Politi, T. Arad, E. Klein, S. Weiner, L. Addadi, Science 2004, 306, $1161-4$.

[6] F. Nudelman, H. H. Chen, H. A. Goldberg, S. Weiner, L. Addadi, Faraday Discuss. 2007, 136, 9-25.

[7] D. Gebauer, K. Jansson, M. Oliveberg, N. Hedin, Minerals 2018, 8, 84.

[8] E. Ruiz-Agudo, A. Burgos-Cara, C. Ruiz-Agudo, A. Ibañez-Velasco, H. Cölfen, C. Rodriguez-Navarro, Nat. Commun. 2017, 8, DOI 10.1038/s41467-017-00756-5

[9] B. Xie, T. J. Halter, B. M. Borah, G. H. Nancollas, Cryst. Growth Des. 2015, 15, 204-211.

[10] A. E. S. Van Driessche, M. Kellermeier, L. G. Benning, D. Gebauer, Eds. , New Perspectives on Mineral Nucleation and Growth, Springer Nature, Cham, Switzerlan, 2017.

[11] Z. Zou, X. Yang, M. Albéric, T. Heil, Q. Wang, B. Pokroy, Y. Politi, L. Bertinetti, Adv. Funct. Mater. 2020, 30, 2000003.

[12] A. Akiva-Tal, S. Kababya, Y. S. Balazs, L. Glazer, A. Berman, A Sagi, A. Schmidt, Proc. Natl. Acad. Sci. 2011, 108, 14763-14768.

[13] L. B. Gower, D. J. D. Odom, J. Cryst. Growth 2000, 210, 719-734.

[14] L. B. Gower, D. Tirrell, J. Cryst. Growth 1998, 191, 153-160.

[15] B. Cantaert, A. Verch, Y.-Y. Kim, H. Ludwig, V. N. Paunov, R. Kro, F. C. Meldrum, R. Kröger, F. C. Meldrum, Chem. Mater. 2013, 25, 4994-5003.

[16] M. Balz, H. A. Therese, J. Li, J. S. Gutmann, M. Kappl, L. Nasdala, W. Hofmeister, H.-J. Butt, W. Tremel, Adv. Funct. Mater. 2005, 15 683-688.

[17] S. J. Homeijer, M. J. Olszta, R. A. Barrett, L. B. Gower, J. Cryst. Growth 2008, 310, 2938-2945.

[18] J. Harris, I. Mey, M. Hajir, M. Mondeshki, S. E. Wolf, CrystEngComm 2015, 17, 6831-6837.

[19] D. E. Rodriguez, T. Thula-Mata, E. J. Toro, Y.-W. Yeh, C. Holt, L. S. Holliday, L. B. Gower, Acta Biomater. 2014, 10, 494-507.

[20] Y.-Y. Kim, N. B. J. Hetherington, E. H. Noel, R. Kröger, J. M. Charnock, H. K. Christenson, F. C. Meldrum, Angew. Chem. Int. Ed. 2011, 50, 12572-12577.

[21] J. Harris, I. P. Mey, C. F. Böhm, T. T. H. Trinh, S. Leupold, C. Prinz, P. Tripal, R. Palmisano, S. E. Wolf, Nanoscale Horizons 2019, 4, 1388-1393.

[22] F. Nudelman, K. Pieterse, A. George, P. H. H. Bomans, H. Friedrich, L. J. Brylka, P. A. J. Hilbers, G. De With, N. A. J. M. Sommerdijk, Nat. Mater. 2010, 9, 9-14.

[23] E. M. Pouget, P. H. H. Bomans, J. A. C. M. Goos, P. M. Frederik, G. de With, N. A. J. M. Sommerdijk, P. H. H. Bomans, J. A. C. M. Goos, P. M. Frederik, G. de With, N. A. J. M. Sommerdijk, Science 2009, 323, 1455-1458.

[24] D. Gebauer, A. Völkel, H. Cölfen, Science 2008, 322, 1819-22.

[25] A. S. A. Mohammed, A. Carino, A. Testino, M. R. Andalibi, A. Cervellino, Part. Part. Syst. Charact. 2019, 36, 1800482.

[26] R. Demichelis, P. Raiteri, J. D. Gale, D. Quigley, D. Gebauer, Nat. Commun. 2011, 2, 590.

[27] R. S. K. Lam, J. M. Charnock, F. C. Meldrum, A. Lennie, F. C. Meldrum, CrystEngComm 2007, 9, 1226-1236.
[28] J. H. E. Cartwright, A. G. Checa, J. D. Gale, D. Gebauer, C. I. Sainz-Díaz, Angew. Chem. Int. Ed. 2012, 51, 11960-70.

[29] S. E. Wolf, L. Müller, R. Barrea, C. J. Kampf, J. Leiterer, U. Panne, T. Hoffmann, F. Emmerling, W. Tremel, Nanoscale 2011, 3, 11581165.

[30] A. R. Finney, P. M. Rodger, Faraday Discuss. 2012, 159, 47

[31] M. Kellermeier, P. Raiteri, J. K. Berg, A. Kempter, J. D. Gale, D. Gebauer, ChemPhysChem 2016, 17, 3535-3541.

[32] F. Sebastiani, S. L. P. Wolf, B. Born, T. Q. Luong, H. Cölfen, D. Gebauer, M. Havenith, Angew. Chem. Int. Ed. 2017, 56, 490-495. D. Gebauer, H. Cölfen, Nano Today 2011, 6, 564-584.

[34] D. Gebauer, M. Kellermeier, J. D. Gale, L. Bergström, H. Cölfen, Chem. Soc. Rev. 2014, 43, 2348-71.

[35] P. J. M. Smeets, A. R. Finney, W. Habraken, F. Nudelman, H. Friedrich, J. Laven, J. J. De Yoreo, P. M. Rodger, N. A. J. M. Sommerdijk, Proc. Natl. Acad. Sci. 2017, 114, E7882-E7890.

[36] J. J. De Yoreo, in Faraday Discuss., 2012.

[37] A. F. Wallace, L. O. Hedges, A. Fernandez-martinez, P. Raiteri, J. D. Gale, G. A. Waychunas, S. Whitelam, J. F. Banfield, J. J. De Yoreo, Science 2013, 341, 885-9.

[38] J. M. Walker, B. Marzec, F. Nudelman, Angew. Chem. Int. Ed. 2017, $56,11740-11743$.

[39] Y. U. T. Gong, C. E. Killian, I. C. Olson, N. P. Appathurai, A. L. Amasino, M. C. Martin, L. J. Holt, F. H. Wilt, P. Gilbert, Proc. Nat. Acad. Sci. USA 2012, 109, 1-6.

[40] C.-Y. Sun, M. A. Marcus, M. J. Frazier, A. J. Giuffre, T. Mass, P. Gilbert, ACS Nano 2017, 11, 6612-6622.

[41] R. T. DeVol, C.-Y. Sun, M. A. Marcus, S. N. Coppersmith, S. C. B. Myneni, P. Gilbert, J. Am. Chem. Soc. 2015, 151007153715000.

[42] S. Kababya, A. Gal, K. Kahil, S. Weiner, L. Addadi, A. Schmidt, J. Am. Chem. Soc. 2015, 137, 990-998.

[43] P. Werner, A. C. S. Jensen, J. Mahamid, R. Dinnebier, P. Fratzl, E. Zolotoyabko, B. Pokroy, S. Weiner, S. Bette, Y. Politi, G. Matveeva, M. A. Hood, W. Habraken, I. Polishchuk, U. Kolb, Z. Zou, C. Sun, P. Gilbert, L. Bertinetti, Science 2019, 363, 396-400.

[44] 2004, Regulation (EC) $N^{\circ} 648 / 2004$ of the European Parliament and of the Council on Detergents, 2004.

[45] D. Gebauer, H. Cölfen, A. Verch, M. Antonietti, Adv. Mater. 2009 21, 435-439.

[46] A. Verch, D. Gebauer, M. Antonietti, H. Cölfen, Phys. Chem. Chem. Phys. 2011, 13, 16811-20.

[47] M. S. Oshchepkov, G. Y. Rudakova, S. V. Tkachenko, V. E. Larchenko, K. I. Popov, M. A. Tusheva, Therm. Eng. 2021, 68, 370380.

[48] M. M. Reddy, A. R. Hoch, J. Colloid Interface Sci. 2001, 235, 365370.

[49] D. J. Tobler, J. D. Rodriguez-Blanco, K. Dideriksen, N. Bovet, K. K. Sand, S. L. S. Stipp, Adv. Funct. Mater. 2015, 25, 3081-3090.

[50] I. Ben Shir, S. Kababya, D. B. Zax, A. Schmidt, J. Am. Chem. Soc. 2020, 142, 13743-13755.

[51] T. Gullion, J. Schaefer, J. Magn. Reson. 1989, 81, 196-200.

[52] D. Gebauer, S. E. Wolf, J. Am. Chem. Soc. 2019, 141, 4490-4504.

[53] S. M. Clark, B. Colas, D. E. Jacob, J. Neuefeind, H.-W. Wang, K. L. Page, A. K. Soper, P. I. Schodder, P. Duchstein, B. Apeleo Zubiri, T. V. P. Yokosawa, D. Zahn, E. Spiecker, S. E. Wolf, ChemRxiv 2021, DOI 10.26434/chemrxiv.14292161.v1.

[54] K. Henzler, E. O. Fetisov, M. Galib, M. D. Baer, B. A. Legg, C. Borca, J. M. Xto, S. Pin, J. L. Fulton, G. K. Schenter, N. Govind, J. I. Siepmann, C. J. Mundy, T. Huthwelker, J. J. De Yoreo, Sci. Adv. 2018, 4, eaao6283.

[55] E. Davies, K. H. Muller, W. C. Wong, C. J. Pickard, D. G. Reid, J. N. Skepper, M. J. Duer, Proc. Natl. Acad. Sci. 2014, 111, E1354E1363.

[56] Y.-Y. Hu, A. Rawal, K. Schmidt-Rohr, Proc. Natl. Acad. Sci. 2010, 107, 22425-22429.

[57] S. J. Omelon, M. D. Grynpas, Chem. Rev. 2008, 108, 4694-4715.

[58] W. E. G. Müller, X. Wang, B. Diehl-Seifert, K. Kropf, U.

Schloßmacher, I. Lieberwirth, G. Glasser, M. Wiens, H. C. Schröder, Acta Biomater. 2011, 7, 2661-2671. 
